\title{
Dark Photon and Muon $g-2$ Inspired Inelastic Dark Matter Models at the High-Energy Intensity Frontier
}

\author{
Yu-Dai Tsai®, ${ }^{1,2,{ }^{*}}$ Patrick deNiverville $\odot,{ }^{3,4, \dagger}$ and Ming Xiong Liu $\oplus^{4, \$}$ \\ ${ }^{1}$ Fermilab, Fermi National Accelerator Laboratory, Batavia, Illinois 60510, USA \\ ${ }^{2}$ Kavli Institute for Cosmological Physics, University of Chicago, Chicago, Illinois 60637, USA \\ ${ }^{3}$ Center for Theoretical Physics of the Universe, IBS, Daejeon 34126, Korea \\ ${ }^{4}$ Los Alamos National Laboratory, Los Alamos, New Mexico 87545, USA
}

(Received 1 January 2020; revised 19 October 2020; accepted 11 March 2021; published 3 May 2021)

We study hidden-sector particles at past (CERN-Hamburg-Amsterdam-Rome-Moscow Collaboration and $\mathrm{NuCal}$ ), present (NA62, SeaQuest, and DarkQuest), and future (LongQuest) experiments at the high-energy intensity frontier. We focus on exploring the minimal vector portal and the next-to-minimal models in which the productions and decays are decoupled. These next-to-minimal models have mostly been devised to explain experimental anomalies while avoiding existing constraints. We demonstrate that proton fixed-target experiments provide one of the most powerful probes for the $\mathrm{MeV}$ to few $\mathrm{GeV}$ mass range of these models, using inelastic dark matter (iDM) as an example. We consider an iDM model with a small mass splitting that yields the observed dark matter relic abundance, and a scenario with a sizable mass splitting that can also explain the muon $g-2$ anomaly. We set strong limits based on the CERN-Hamburg-Amsterdam-RomeMoscow Collaboration and $\mathrm{NuCal}$ experiments, which come close to excluding iDM as a full-abundance thermal dark matter candidate in the $\mathrm{MeV}$ to $\mathrm{GeV}$ mass range. We also make projections based on NA62, SeaQuest, and DarkQuest and update the constraints of the minimal dark photon parameter space. We find that $\mathrm{NuCal}$ sets the only existing constraint in $\epsilon \sim 10^{-8}-10^{-4}$ regime, reaching $\sim 800 \mathrm{MeV}$ in dark photon mass due to the resonant enhancement of proton bremsstrahlung production. These studies also motivate LongQuest, a three-stage retooling of the SeaQuest experiment with short $(\lesssim 5 \mathrm{~m})$, medium $(\sim 5 \mathrm{~m})$, and long ( $\approx 35 \mathrm{~m}$ ) baseline tracking stations and detectors as a multipurpose machine to explore new physics.

DOI: 10.1103/PhysRevLett.126.181801

Introduction.-Given the nonobservation of new physics at the $\mathrm{TeV}$ scale and the ever-stronger constraints on weakly interacting massive particle dark matter (DM) [1], many physicists have shifted their attention to the study of dark-sector particles with sub-GeV to few $\mathrm{GeV}$ masses. This regime is also of great interest due to a number of experimental anomalies, including the muon $g-2$ anomaly $[2,3]$ and the Land Scintillator Neutrino Detector and Mini Booster Neutrino Experiment excesses [4,5].

Proton fixed-target machines are among the most powerful and robust probes of dark-sector and long-lived particles in the $\mathrm{MeV}$ to $\sim 10 \mathrm{GeV}$ regime (e.g., [6-18]). First, the searches benefit from the combination of high energy and high intensity that the proton beams provide. Second, these accelerator-based probes do not depend on DM abundance, velocity distribution, or cosmic history. The constraints

Published by the American Physical Society under the terms of the Creative Commons Attribution 4.0 International license. Further distribution of this work must maintain attribution to the author(s) and the published article's title, journal citation, and DOI. Funded by SCOAP ${ }^{3}$. and sensitivity reach also do not depend on complicated astrophysics or rare events. As our theoretical understanding of the dark sector advances, it is crucial to look back at the highest-energy experiments of the intensity frontier. These include past experiments like the CERN-HamburgAmsterdam-Rome-Moscow Collaboration (CHARM) and $\mathrm{NuCal}(\nu$-Cal I) and ongoing experiments like NA62, SeaQuest, and SpinQuest and their beam-dump and upgrade proposals (the NA62 beam-dump run and the DarkQuest proposal [19]). It is also important to look closely at the existing facilities to see if low-cost repurposing or upgrades can help further explore beyond-thestandard-model physics [20,21].

To demonstrate the strength of proton fixed-target experiments, we first revisit several of the existing constraints of the vector portal $[8,11,22-81]$, fill in the missing relevant production channels, and conduct a robust reanalysis based on the available data. Furthermore, we study a next-tominimal class of models considered to have a certain range of lifetimes or decay lengths to avoid experimental or observational bounds while explaining specific anomalies. One of the best examples of this class of models is the inelastic dark matter (iDM) model [10,82-87]. In the case of iDM, the production of the dark-sector particles is 
predominantly through small standard model (SM) coupling, but the decay length of the dark-sector particles in the search is also determined by the mass splitting of nearly degenerate dark particle states. iDM provides one of the few viable $\mathrm{GeV}$ and sub-GeV thermal DM scenarios that freezes out to the right relic abundance, also called "thermal targets" (thermal DM that can be probed in near-future experiments) [88-103]. iDM was also studied as one of the last viable vector-portal explanations of the muon $g-2$ anomaly $[2,86,104]$.

We set strong new constraints on iDM model parameters in the $\mathrm{MeV}$ to $\mathrm{GeV}$ regime, based on CHARM and $\mathrm{NuCal}$ beam-dump experiments, for both small and sizable iDM mass splitting, ruling out a large portion of the parameter space for iDM to account for the total DM abundance. We also revisit and improve the minimal dark photon bounds set by the pioneering analyses $[51,53,54]$. In addition, we perform the first dark photon and iDM sensitivity projections based on the NA62 beam-dump run. NA62 can further extend the sensitivity of the two scenarios and help to close the window of parameter space for which iDM models can explain the muon $g-2$ anomaly. Finally, we conduct a study of iDM in the muon $g-2$ regime based on the DarkQuest upgrade of the SeaQuest experiment $[8,13,105]$. We demonstrate a general point that high-energy proton fixed-target machines provide strong probes of models that would otherwise escape the bounds from other experiments.

Inspired by the studies of SeaQuest and DarkQuest (SQ-DQ), we consider a complete retooling of the SeaQuest and SpinQuest experiments as a dedicated and multipurpose experiment to conduct dark-sector searches with short $(\lesssim 5 \mathrm{~m})$, medium $(\sim 5-12 \mathrm{~m})$, and long $(\gtrsim 35 \mathrm{~m})$ baseline detectors. We designate all these new installations as "LongQuest" and discuss the three-stage retooling in the Supplemental Material [106].

Models and signatures.—Dark photon from kinetic mixing: A minimal model of a visibly decaying dark photon through the kinetic mixing between SM $U(1)_{Y}$ and a dark $U(1)$ gauge field is given by

$$
\mathcal{L}_{\text {kinmix }}=\frac{\epsilon}{2 \cos \theta_{W}} A_{\mu \nu}^{\prime} B^{\mu \nu},
$$

where $\theta_{W}$ is the Weinberg angle. The crucial interaction term between dark photon and SM particles can be expressed as

$$
\mathcal{L}_{\text {int }} \supset \epsilon e A_{\mu}^{\prime} \mathcal{J}_{\mathrm{EM}}^{\mu},
$$

where $\mathcal{J}_{\mathrm{EM}}^{\mu}$ is the SM electromagnetic current. We consider the case of a massive $A^{\prime}$ where the mass $m_{A^{\prime}}$ can be generated through the Higgs or Stueckelberg mechanisms.

There are three main production channels considered in the literature for kinetically mixed dark photons: meson decays, proton bremsstrahlung, and Drell-Yan and QCD processes. Each of these processes dominates dark photon production for different dark photon masses. Among these, the Drell-Yan and QCD productions suffer large uncertainties in the sub-GeV energy regime given the uncertainty in the parton distribution functions [77,107] and thus were not included in our analysis. All the other relevant production processes were considered in our analysis.

Inelastic DM: Here, we study iDM composed of a Dirac pair of two-component Weyl spinors, $\eta$ and $\xi$, charged under a new $U(1)_{D}$ gauge symmetry. We include the DM interaction term as

$$
\mathcal{L}_{\text {int }} \supset \epsilon e A_{\mu}^{\prime} \mathcal{J}_{\mathrm{EM}}^{\mu}+g_{D} A_{\mu}^{\prime} \mathcal{J}_{\mathcal{D}}^{\mu}
$$

$\mathcal{J}_{\mathcal{D}}^{\mu}$ is the dark-sector current to which the dark photon $A^{\prime}$ couples. We express $g_{D} \equiv \sqrt{4 \pi \alpha_{D}}$. This dark-sector current consists of a four-component fermionic state $\Psi=\left(\eta \xi^{\dagger}\right)$ with two-component Weyl spinors $\eta$ and $\xi$. Again, $A^{\prime}$ is massive and $U(1)_{D}$ is broken, and one can write down the Majorana and Dirac mass terms as

$$
\mathcal{L} \supset-m_{D} \eta \xi-\frac{1}{2} \delta_{\eta} \eta^{2}-\frac{1}{2} \delta_{\xi} \xi^{2}+\text { H.c. }
$$

$\delta_{\eta}, \delta_{\xi} \ll m_{D}$ are technically natural because they break the $U(1)$ symmetry explicitly. After the mass diagonalization $m_{12} \simeq m_{D} \mp \frac{1}{2}\left(\delta_{\eta}+\delta_{\xi}\right)$, we have $\chi_{1} \simeq i(\eta-\xi) / \sqrt{2}, \chi_{2} \simeq$ $(\eta+\xi) / \sqrt{2}$.

The relevant parts of the Lagrangian in terms of the mass eigenstates $\chi_{1}$ and $\chi_{2}$ are

$$
\mathcal{L} \supset \sum_{i=1,2} \bar{\chi}_{i}\left(i \not \partial-m_{\chi_{i}}\right) \chi_{i}-\left(g_{D} A_{\mu}^{\prime} \overline{\chi_{1}} \gamma^{\mu} \chi_{2}+\text { H.c. }\right) .
$$

The elastic interactions are suppressed by a factor of $\delta / m_{D}$. $\delta \ll m_{D}$ is again technically natural because the $U(1)$ explicit breaking would be restored when $\delta \rightarrow 0$. Note that the elastic interaction vanishes as $\delta_{\eta}=\delta_{\xi}$.

The particle $\chi_{1}$, which we take to be lighter than $\chi_{2}$, can account for the current-day DM abundance. The mass splitting is defined as

$$
\Delta \equiv \frac{m_{2}-m_{1}}{m_{1}}
$$

An approximate analytical expression for the $A^{\prime}$ dominantly decays to $\chi_{1} \chi_{2}$ when the mass splitting $\Delta$ and the elastic coupling terms are small is

$$
\Gamma\left(A^{\prime} \rightarrow \chi_{1} \chi_{2}\right) \simeq \frac{\alpha_{D} m_{A^{\prime}}}{3} \sqrt{1-\frac{4 m_{1}^{2}}{m_{A^{\prime}}^{2}}}\left(1+\frac{2 m_{1}^{2}}{m_{A^{\prime}}^{2}}\right) .
$$

An approximation for the width of the $\chi_{2}$ decay also exists in the small $\Delta$ limit when $m_{A^{\prime}} \gg m_{1} \gg m_{l}$ : 


$$
\Gamma\left(\chi_{2} \rightarrow \chi_{1} l^{+} l^{-}\right) \simeq \frac{4 \epsilon^{2} \alpha_{\mathrm{em}} \alpha_{D} \Delta^{5} m_{1}^{5}}{15 \pi m_{A^{\prime}}^{4}} .
$$

We would like to emphasize that, in our analysis, we simulated the three-body decay $\chi_{2} \rightarrow e^{+} e^{-} \chi_{1}$ by sampling the full decay width, calculated in the Supplemental Material [106], rather than using the approximations here.

The dark photon has been proposed as an explanation of the muon $g-2$ anomaly [2,104,108-117]. The minimal models assume that the dark photon either decays completely visibly [30,39-41,56,62] or invisibly to DM particles $[88,108]$ and are excluded by various experiments when they assume large enough kinetic mixing to account for the muon $g-2$ anomaly $[62,118,119]$. These constraints can be weakened if the dark photon is allowed to decay semivisibly, as is possible in iDM models [86]. If $\Delta$ is sufficiently large, the $\chi_{2}$ will decay inside the detector and thus avoid the invisible decay bounds on elastic DM [118-120].

iDM thermal target and muon $g-2$ window: The search strategy in this Letter is to look for the decay of the dark photon through $A^{\prime} \rightarrow \ell^{+} \ell^{-}$or the three-body semivisible decay $\chi_{2} \rightarrow \chi_{1} \ell^{+} \ell^{-}$, described in detail in the Supplemental Material [106]. The decay signature is a lepton-antilepton pair, and the best probes are "decay detectors" in a fixed-target setup. In this addition, we discuss each of the experiments considered in detailincluding CHARM, NA62, NuCal and U70, and SeaQuest and DarkQuest—in the Supplemental Material [106] and summarize some useful experimental information for comparison in Table I of the Supplemental Material [106].

One of the main goals of this Letter is to provide constraints on the canonical iDM with a small mass splitting: $\Delta \ll 1$. We consider fermionic iDM and fix the mediator to DM mass ratio to be $m_{A^{\prime}}=3 m_{1}$. In the small mass-splitting regime, we consider fractional mass differences $\Delta=0.1$ with $\alpha_{D}=0.1$ and $\Delta=0.05$ with $\alpha_{D}=0.5$ as examples. Also, we fix the value of the kinetic mixing $\epsilon$, while varying $\alpha_{D}$ such that the model produces the observed relic abundance. We show the new constraints and projections for the small mass splitting, for $\Delta=0.1$ with $\alpha_{D}=0.1 \mathrm{iDM}$ parameter space, in Fig. 2 and the results of other choices of parameters in the Supplemental Material [106].

In addition, we present a detailed discussion of the sensitivity of proton-beam fixed-target facilities to study iDM with $\Delta \gtrsim 0.4$ comprising a parameter regime that could explain the muon $g-2$ anomaly and avoid other experimental constraints. The existing data from CHARM and $\mathrm{NuCal}$ are considered, and we also include studies based on the NA62 beam-dump mode as well as the SQDQ configuration. We consider iDM with a sizable masssplitting regime, $\Delta=0.4$, and coupling $\alpha_{D}=0.1$. Then, we fix the $\epsilon$ to the value that gives the correct muon $g-2$ value while varying $\alpha_{D}$ to compare the current constraints and future sensitivity reach. The results are shown in Fig. 3. Although strong constraints can be set, the muon $g-2$

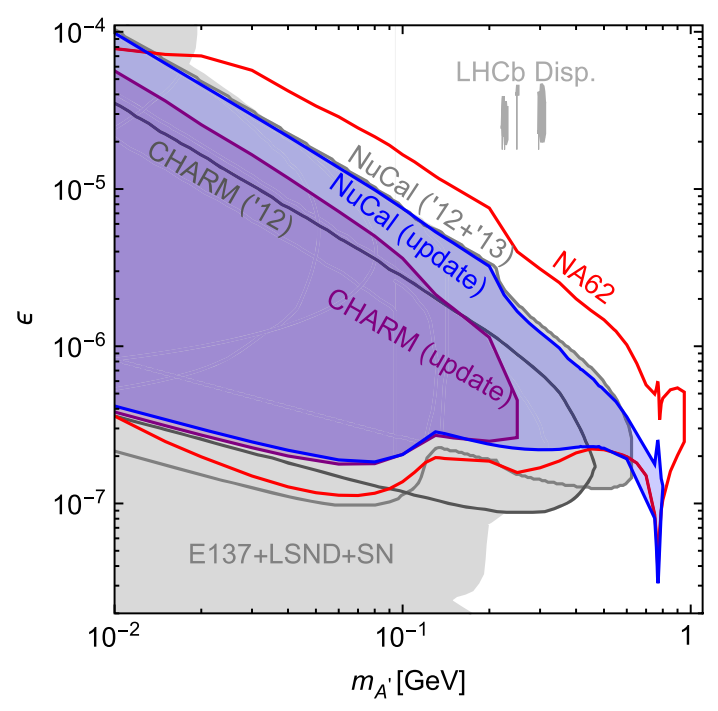

(a)

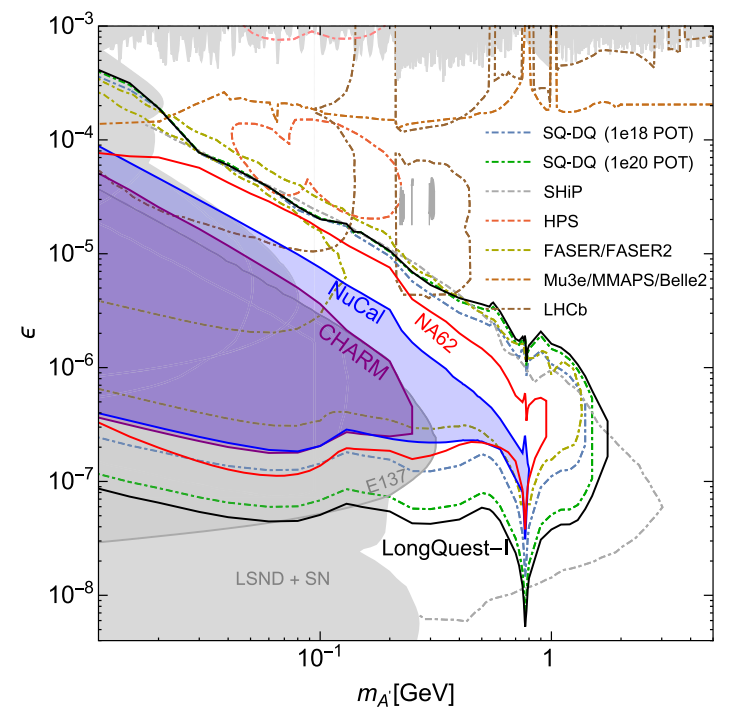

(b)

FIG. 1. We show updates on the kinetically mixed visibly decaying dark photon constraints and projections. In (a), gray contours are the previous bounds set based on analyses of the $\mathrm{NuCal}[51,54]$ and CHARM [53] experiments. In (b), projections for future experiments are shown in dot-dashed curves with color and also labeled in the plot. In both (a) and (b), our updated bounds on CHARM (purple) and NuCal (blue) and our new projections of NA62 (red) and LongQuest-I (black) are shown. (a) Updates on dark photon bounds and the NA62 projection. (b) Compilation of projections and constraints on dark photon.

target regime cannot be excluded because the distances between the targets and the decay regions are too large, and $\chi_{2}$ decays before reaching the fiducial detector region. This provides a motivation to consider the LongQuest upgrade of SQ-DQ to probe this regime.

Results and discussions.-Minimal dark photon: We update the dark photon bounds from CHARM and 
NuCal in Fig. 1(a), taking into account additional relevant production channels (production of dark photons from $\eta$ meson decays for $\mathrm{NuCal}$ and proton bremsstrahlung for CHARM). Interestingly, the bound does not get stronger for most parameter spaces in our consideration, except for the high mass regime, even though we take into account new relevant production channels. This is because the strength of the bounds in the meson-decay dominated regime $\left(m_{A^{\prime}} \ll m_{\eta}\right)$ are highly dependent on the estimate of the overall meson production rate, and ours are conservative (see Ref. [121] for comparisons between different methods of estimating $\pi^{0}$ production).

At large masses, there is an improvement from our handling of the proton bremsstrahlung, which considers a timelike form factor $[9,131]$ with resonant enhancement from mixing with the $\rho$ and $\omega$ mesons, visible in the constraint plots as a sharp peak. The NuCal contour is also the first constraint of this kind with a dark photon mass reach close to $1 \mathrm{GeV}$, as shown in Fig. 1. CHARM's production is not enhanced to the same extent due in part to its off-axis position and the highly collimated nature of bremsstrahlung. We also studied NA62's projected sensitivity in its proposed beam-dump mode based on [121] and found that it could explore stronger couplings due in large part to the higher energy beam and that the same $\rho$ peak exhibited by NuCal appears at larger masses.

Finally, we make projections for the sensitivity of SQ-DQ and LongQuest-I with a simplified detector simulation. The short baseline relative to the length of the decay volume renders the detector particularly sensitive to the lifetime of the dark photon, which combined with the SeaqQuest detector simulation resulted in some statistical noise in the sensitivity. In Fig. 1(b), we show the sensitivity

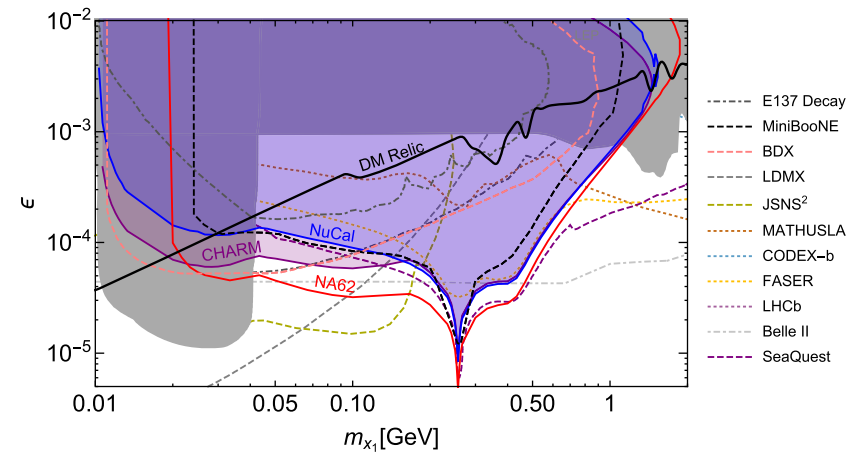

FIG. 2. We show new constraints on iDM based on the data of CHARM (purple) and NuCal (blue), and the projected sensitivity of the NA62 beam-dump run (red). The gray shaded regions are previously existing constraints (see Sec. IB). We also include the potential E137 decay constraint $[10,13,40]$ along with future projections $[10,13,32,73,76,77,85,122-130]$. One can see the E137, Mini Booster Neutrino Experiment, and Beam Dump Experiment (BDX) projections are already covered by CHARM and NuCal. (a) Compilation of constraints and sensitivity projections for iDM with $m_{A^{\prime}}=3 m_{1}, \alpha_{D}=0.1$, and $\Delta=0.1$. projections of the $10^{20}$ protons on target (POT) runs for SQ-DQ and LongQuest-I. We demonstrate the potential advantage of such an improved decay detector and the reduction of background rate to a $10 \%$ level by comparing the SQ-DQ and LongQuest-I curves.

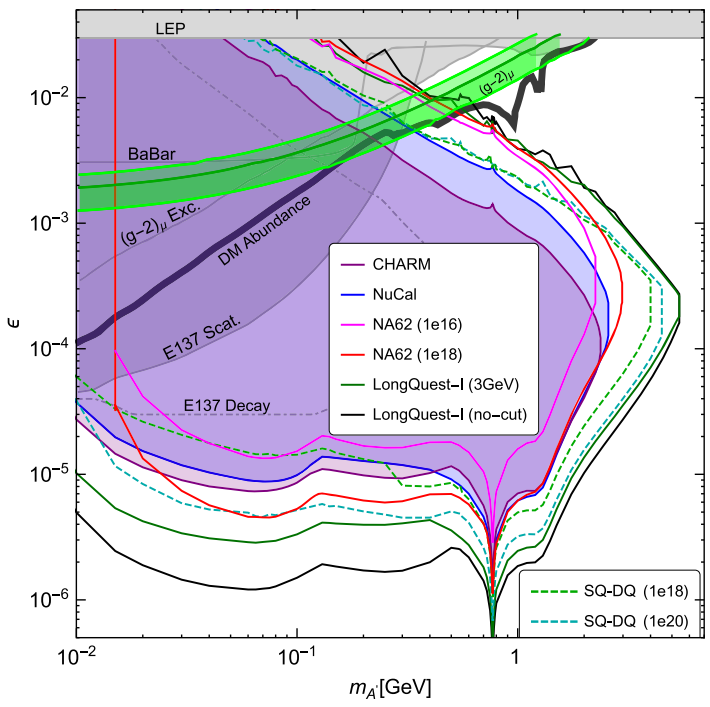

(a)

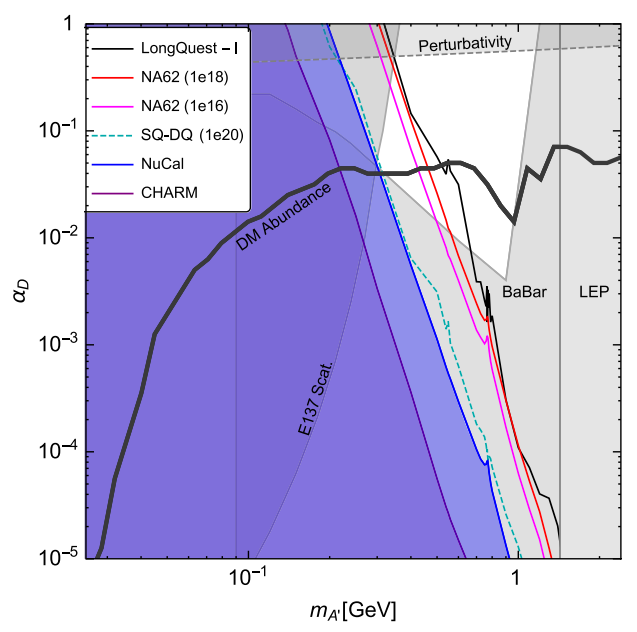

(b)

FIG. 3. These plots show constraints and sensitivity projections for iDM within the muon $g-2$ motivated regime. Here we consider $m_{A^{\prime}}=3 m_{1}$ for demonstration. The muon $g-2$ favored regime is the light-green band in (a), while the thick-black curves are again the parameter contour yielding the correct DM relic abundance. We considered the bounds from CHARM (purple) and $\mathrm{NuCal}$ (blue) and projections from NA62 [10 ${ }^{18}$ POT (red), $1.3 \times 10^{16}$ POT (magenta)], SQ-DQ [10 ${ }^{20}$ POT (dashed-cyan), $1.4 \times 10^{18}$ POT (dashed-pine green), and LongQuest-I (3-GeV cut: darker green; no-cut: black)]. In (b), we only plot the LongQuest-I no cut curve because it is basically identical to the $3-\mathrm{GeV}$ cut result. The gray region denotes the previously existing constraints. (a) iDM: $\Delta=0.4, \alpha_{D}=0.1$. With muon $g-2$ and DM regimes. (b) iDM Muon $g-2$ Target: $\Delta=0.5$, $\epsilon=\epsilon_{(g-2)_{\mu}}$. 
iDM thermal and $(g-2)_{\mu}$ targets: The constraints and sensitivity projections for iDM with $\Delta=0.1, \alpha=0.1$ are shown in Fig. 2 and the parameter space to explain the muon $g-2$ anomaly in Fig. 3. Other iDM constraints and projections are plotted in Fig. 6 of the Supplemental Material [106]. All previously considered experimental constraints in this parameter space are included and labeled in the plots $[6,13,40,73,119,132-137]$, with the exception of the potential constraints from the recast of the E137 analysis [40], discussed in the Supplemental Material [106]. Below $10 \mathrm{MeV}$, the iDM model is subject to bounds on $N_{\text {eff }}[138,139]$, and the proton fixed-target probes become suppressed by the limited phase space available to the $\chi_{1} l^{+} l^{-}$states. We therefore choose to focus on iDM masses larger than $10 \mathrm{MeV}$.

As shown in Fig. 2, CHARM and NuCal provide strong constraints on iDM with small mass splittings, excluding almost all of the regimes that predict the correct DM relic abundance. NA62 can further improve the exploration of these iDM scenarios. Other future probes of iDM $[10,13,32,73,76,77,85,122-130]$ relevant for the regime of interest are also included in Fig. 2. The benchmark thermal relic curve we consider in Fig. 2 of relic iDM is already ruled out by previous experiments. In the Supplemental Material [106], we show that there is still a small region of parameter space when the kinetic mixing and masses are small in the small mass-splitting case (e.g., $\delta=0.05$ is picked to demonstrate that), in when that the relic abundance line survives current constraints.

For the large mass-splitting iDM parameter space motivated by the muon $g-2$ anomaly, proton fixed-target experiments provide strong new constraints, as shown in Fig. 3. However, in order to resolve the muon $g-2$ discrepancy, there must be large kinetic mixing between the dark and SM photons, and the dark photon must have a mass $\gtrsim 300 \mathrm{MeV}$ to escape existing constraints. The CHARM and NuCal analyses poorly constrain the open muon $g-2$ favored parameter space because the large mass and strong coupling to the SM leads the $\chi_{2}$ particles to decay before they reach the decay regions.

In Fig. 3, we also show the first study of the iDM muon $g-2$ regime in SQ-DQ assuming a sizable mass splitting: $\Delta=0.4$. We place a contour on more than ten events for the sensitivity of SQ-DQ $10^{18}$ POT run. For the SQ-DQ $10^{20}$ POT run, we assume $10^{3}$ background events (a rough estimation from long-lived kaon decays) to set a sensitivity projection. One can see that SQ-DQ does improve the sensitivity to smaller couplings with a $10^{20}$ POT run. However, as can be seen in this figure, the sensitivity to iDM in the $(g-2)_{\mu}$ valid regime is not improved by SQDQ phase I and II.

In principle, SQ-DQ should provide the best sensitivity probes in this regime, given that it has the shortest distance between the target and the fiducial decay region among all the experiments we consider. However, we found that the sensitivity to this regime is only comparable to that of $\mathrm{CHARM}$ and $\mathrm{NuCal}$ because the strong magnetic field in the KMAG suppresses new physics events by kicking the visible charged products out of the decay volume [13]. This effect is particularly significant for iDM because the lepton pairs from $\chi_{2}$ decays are soft, given that the $\chi_{1}$ takes a large fraction of the $\chi_{2}$ energy. The signal suppression from the KMAG effectively cancels out the benefit of a shorter baseline of SQ-DQ.

Finally, we show that the proposed LongQuest-I upgrades with reduced background and no KMAG magnetic field would help explore the iDM muon g-2 regime in Fig. 3. For the LongQuest-I analysis, we assume the background is reduced to $10 \%$ of the SQ-DQ background. Given the removal of KMAG and the potential soft SM background, we consider the sensitivity of a 3-GeV energy threshold cut and a projection with no energy threshold cut. Further discussions can be found in the Supplemental Material [106], which also includes Refs. [6-9,11,12,14, 17-20,40,43,44,51,53,54,71,80,86,105,109,121,122,137, 140-161]. A realistic analysis may need to apply an energy cut between these choices.

We thank Asher Berlin and Gopolang Mohlabeng for helpful correspondence. We thank Paddy Fox, Kun Liu, and Sho Uemura for the discussion of the SeaQuest and DarkQuest experimental configurations. We thank Peter Cooper, Babette Dobrich, and Gaia Lanfranchi for useful discussions regarding the NA62 experiment. We thank Carlos Arguelles, Nikita Blinov, Iftah Galon, Matheus Hostert, Gordan Krnjaic, and Seodong Shin for useful discussions. The authors thank APCTP (Korea) and TRIUMF (Canada) for stimulating dark matter workshops. Y.-D. T. sincerely thanks CERN, where this work was initiated, and its theory group for the hospitality. Parts of the work was performed at the Aspen Center for Physics, which is supported by National Science Foundation Grant No. PHY-1607611. The work of P. D. was supported by IBS (Project Code IBS-R018-D1) and by Los Alamos National Laboratory under the LDRD program. M.L. thanks the DOE Office of Science, Nuclear Physics and is supported by Los Alamos National Laboratory LDRD 20160081ER. This manuscript has been authored by Fermi Research Alliance, LLC under Contract No. DE-AC02-07CH11359 with the U.S. Department of Energy, Office of Science, Office of High Energy Physics. The publisher, by accepting the article for publication, acknowledges that the U.S. Government retains a nonexclusive, paid-up, irrevocable, worldwide license to publish or reproduce the published form of this manuscript, or allow others to do so, for U.S. Government purposes.

\footnotetext{
* Corresponding author.

ytsai@fnal.gov

Corresponding author.

pgdeniverville@gmail.com
} 
${ }^{\ddagger}$ Corresponding author. ming@bnl.gov

[1] G. Jungman, M. Kamionkowski, and K. Griest, Supersymmetric dark matter, Phys. Rep. 267, 195 (1996).

[2] G. W. Bennett et al. (Muon g-2 Collaboration), Final report of the muon E821 anomalous magnetic moment measurement at BNL, Phys. Rev. D 73, 072003 (2006).

[3] B. Abi et al. (Muon g-2 Collaboration), Measurement of the Positive Muon Anomalous Magnetic Moment to 0.46 ppm, Phys. Rev. Lett. 126, 141801 (2021).

[4] C. Athanassopoulos et al. (LSND Collaboration), Candidate Events in a Search for $\bar{\nu}_{\mu} \rightarrow \bar{\nu}_{e}$ Oscillations, Phys. Rev. Lett. 75, 2650 (1995).

[5] A. A. Aguilar-Arevalo et al. (MiniBooNE Collaboration), Observation of a Significant Excess of Electron-Like Events in the MiniBooNE Short-Baseline Neutrino Experiment, Phys. Rev. Lett. 121, 221801 (2018).

[6] P. deNiverville, M. Pospelov, and A. Ritz, Observing a light dark matter beam with neutrino experiments, Phys. Rev. D 84, 075020 (2011).

[7] Y. Kahn, G. Krnjaic, J. Thaler, and M. Toups, DAE $\delta$ ALUS and dark matter detection, Phys. Rev. D 91, 055006 (2015).

[8] S. Gardner, R. J. Holt, and A. S. Tadepalli, New prospects in fixed target searches for dark forces with the SeaQuest experiment at Fermilab, Phys. Rev. D 93, 115015 (2016).

[9] P. deNiverville, C.-Y. Chen, M. Pospelov, and A. Ritz, Light dark matter in neutrino beams: Production modelling and scattering signatures at MiniBooNE, T2K and SHiP, Phys. Rev. D 95, 035006 (2017).

[10] E. Izaguirre, Y. Kahn, G. Krnjaic, and M. Moschella, Testing light dark matter coannihilation with fixed-target experiments, Phys. Rev. D 96, 055007 (2017).

[11] M. Pospelov and Y.-D. Tsai, Light scalars and dark photons in Borexino and LSND experiments, Phys. Lett. B 785, 288 (2018).

[12] G. Magill, R. Plestid, M. Pospelov, and Yu-D. Tsai, Dipole portal to heavy neutral leptons, Phys. Rev. D 98, 115015 (2018).

[13] A. Berlin, S. Gori, P. Schuster, and N. Toro, Dark sectors at the Fermilab SeaQuest experiment, Phys. Rev. D 98, 035011 (2018).

[14] G. Magill, R. Plestid, M. Pospelov, and Yu-D. Tsai, Millicharged Particles in Neutrino Experiments, Phys. Rev. Lett. 122, 071801 (2019).

[15] E. Bertuzzo, S. Jana, P. A. N. Machado, and R. Zukanovich Funchal, Dark Neutrino Portal to Explain MiniBooNE Excess, Phys. Rev. Lett. 121, 241801 (2018).

[16] P. Ballett, S. Pascoli, and M. Ross-Lonergan, U(1)' mediated decays of heavy sterile neutrinos in MiniBooNE, Phys. Rev. D 99, 071701(R) (2019).

[17] C. A. Argüelles, M. Hostert, and Y.-D. Tsai, Testing New Physics Explanations of MiniBooNE Anomaly at Neutrino Scattering Experiments, Phys. Rev. Lett. 123, 261801 (2019).

[18] C. A. Argüelles et al., White paper on new opportunities at the next-generation neutrino experiments (Part 1: BSM neutrino physics and dark matter), Rep. Prog. Phys. 83, 124201 (2020).
[19] M. X. Liu, Prospects of direct search for dark photon and dark Higgs in SeaQuest/E1067 experiment at the Fermilab main injector, Mod. Phys. Lett. A 32, 1730008 (2017).

[20] K. J. Kelly and Y.-D. Tsai, Proton fixed-target scintillation experiment to search for minicharged particles, Phys. Rev. D 100, 015043 (2019).

[21] S. Foroughi-Abari, F. Kling, and Y.-D. Tsai, FORMOSA: Looking forward to millicharged dark sectors, arXiv :2010.07941.

[22] L. B. Okun, Limits of electrodynamcis: Paraphotons?, Zh. Eksp. Teor. Fiz. 83, 892 (1982) [Sov. Phys. JETP 56, 502 (1982)].

[23] P. Galison and A. Manohar, Two Z's or not two Z's?, Phys. Lett. 136B, 279 (1984).

[24] B. Holdom, Two U(1)'s and $\epsilon$ charge shifts, Phys. Lett. 166B, 196 (1986).

[25] M. Pospelov, A. Ritz, and M. B. Voloshin, Secluded WIMP dark matter, Phys. Lett. B 662, 53 (2008).

[26] N. Arkani-Hamed, D. P. Finkbeiner, T. R. Slatyer, and N. Weiner, A theory of dark matter, Phys. Rev. D 79, 015014 (2009).

[27] J. D. Bjorken, R. Essig, P. Schuster, and N. Toro, New fixed-target experiments to search for dark gauge forces, Phys. Rev. D 80, 075018 (2009).

[28] B. Aubert et al. (BABAR Collaboration), Search for Dimuon Decays of a Light Scalar Boson in Radiative Transitions $\Upsilon \rightarrow \gamma A^{0}$, Phys. Rev. Lett. 103, 081803 (2009).

[29] D. Curtin et al., Exotic decays of the $125 \mathrm{GeV}$ Higgs boson, Phys. Rev. D 90, 075004 (2014).

[30] J. P. Lees et al. (BABAR Collaboration), Search for a Dark Photon in $e^{+} e^{-}$Collisions at BABAR, Phys. Rev. Lett. 113, 201801 (2014).

[31] M. Ablikim et al. (BESIII Collaboration), Dark photon search in the mass range between 1.5 and $3.4 \mathrm{GeV} / c^{2}$, Phys. Lett. B 774, 252 (2017).

[32] R. Aaij et al. (LHCb Collaboration), Search for Dark Photons Produced in $13 \mathrm{TeV} p p$ Collisions, Phys. Rev. Lett. 120, 061801 (2018).

[33] F. Archilli et al. (KLOE-2 Collaboration), Search for a vector gauge boson in $\phi$ meson decays with the KLOE detector, Phys. Lett. B 706, 251 (2012).

[34] A. Anastasi et al. (KLOE-2 Collaboration), Limit on the production of a new vector boson in $e^{+} e^{-} \rightarrow U \gamma, \mathrm{U} \rightarrow$ $\pi^{+} \pi^{-}$with the KLOE experiment, Phys. Lett. B 757, 356 (2016).

[35] F. Bergsma et al. (CHARM Collaboration), A search for decays of heavy neutrinos in the mass range $0.5 \mathrm{GeV}$ to $2.8 \mathrm{GeV}$, Phys. Lett. 166B, 473 (1986).

[36] F. Bergsma et al. (CHARM Collaboration), Search for axion like particle production in $400-\mathrm{GeV}$ proton-Copper interactions, Phys. Lett. 157B, 458 (1985).

[37] G. Bernardi et al., Search for neutrino decay, Phys. Lett. 166B, 479 (1986).

[38] A. Konaka et al., Search for Neutral Particles in ElectronBeam-Dump Experiment, Phys. Rev. Lett. 57, 659 (1986).

[39] E. M. Riordan et al., Search for Short-Lived Axions in an Electron-Beam-Dump Experiment, Phys. Rev. Lett. 59, 755 (1987). 
[40] J. D. Bjorken, S. Ecklund, W. R. Nelson, A. Abashian, C. Church, B. Lu, L. W. Mo, T. A. Nunamaker, and P. Rassmann, Search for neutral metastable penetrating particles produced in the SLAC beam dump, Phys. Rev. D 38, 3375 (1988).

[41] A. Bross, M. Crisler, S. H. Pordes, J. Volk, S. Errede, and J. Wrbanek, A Search for Short-Lived Particles Produced in an Electron Beam Dump, Phys. Rev. Lett. 67, 2942 (1991).

[42] M. Davier and H. Nguyen Ngoc, An unambiguous search for a light Higgs boson, Phys. Lett. B 229, 150 (1989).

[43] J. Blümlein et al., Limits on neutral light scalar and pseudoscalar particles in a proton beam dump experiment, Z. Phys. C 51, 341 (1991).

[44] J. Blümlein et al., Limits on the mass of light (pseudo) scalar particles from Bethe-Heitler $e^{+} e^{-}$and $\mu^{+} \mu^{-}$pair production in a proton-iron beam dump experiment, Int. J. Mod. Phys. A 07, 3835 (1992).

[45] C. Athanassopoulos et al. (LSND Collaboration), Evidence for $\nu_{\mu} \rightarrow \nu_{e}$ oscillations from pion decay in flight neutrinos, Phys. Rev. C 58, 2489 (1998).

[46] P. Astier et al. (NOMAD Collaboration), Search for heavy neutrinos mixing with tau neutrinos, Phys. Lett. B 506, 27 (2001).

[47] R. Essig, R. Harnik, J. Kaplan, and N. Toro, Discovering new light states at neutrino experiments, Phys. Rev. D 82, 113008 (2010).

[48] M. Williams, C. P. Burgess, A. Maharana, and F. Quevedo, New constraints (and motivations) for Abelian gauge bosons in the MeV-TeV mass range, J. High Energy Phys. 08 (2011) 106.

[49] S. N. Gninenko, Stringent limits on the $\pi^{0} \rightarrow \gamma X, X \rightarrow$ $e^{+} e^{-}$decay from neutrino experiments and constraints on new light gauge bosons, Phys. Rev. D 85, 055027 (2012).

[50] S. Abrahamyan et al. (APEX Collaboration), Search for a New Gauge Boson in Electron-Nucleus Fixed-Target Scattering by the APEX Experiment, Phys. Rev. Lett. 107, 191804 (2011).

[51] J. Blumlein and J. Brunner, New exclusion limits for dark gauge forces from beam-dump data, Phys. Lett. B 701, 155 (2011).

[52] H. Merkel et al. (A1 Collaboration), Search for Light Gauge Bosons of the Dark Sector at the Mainz Microtron, Phys. Rev. Lett. 106, 251802 (2011).

[53] S. N. Gninenko, Constraints on sub-GeV hidden sector gauge bosons from a search for heavy neutrino decays, Phys. Lett. B 713, 244 (2012).

[54] J. Blümlein and Jürgen Brunner, New exclusion limits on dark gauge forces from proton bremsstrahlung in beamdump data, Phys. Lett. B 731, 320 (2014).

[55] S. Andreas, C. Niebuhr, and A. Ringwald, New limits on hidden photons from past electron beam dumps, Phys. Rev. D 86, 095019 (2012).

[56] H. Merkel et al. (A1 Collaboration), Search at the Mainz Microtron for Light Massive Gauge Bosons Relevant for the Muon g-2 Anomaly, Phys. Rev. Lett. 112, 221802 (2014).

[57] R. Meijer Drees et al. (SINDRUM I Collaboration), Search for Weakly Interacting Neutral Bosons Produced in $\pi^{-} p$ Interactions at Rest and Decaying into $e^{+} e^{-}$Pairs, Phys. Rev. Lett. 68, 3845 (1992).
[58] D. Babusci et al. (KLOE-2 Collaboration), Limit on the production of a light vector gauge boson in $\phi$ meson decays with the KLOE detector, Phys. Lett. B 720, 111 (2013).

[59] P. Adlarson et al. (WASA-at-COSY Collaboration), Search for a dark photon in the $\pi^{0} \rightarrow e^{+} e^{-} \gamma$ decay, Phys. Lett. B 726, 187 (2013).

[60] G. Agakishiev et al. (HADES Collaboration), Searching for a dark photon with HADES, Phys. Lett. B 731, 265 (2014).

[61] A. Adare et al. (PHENIX Collaboration), Search for dark photons from neutral meson decays in $p p$ and $d \mathrm{Au}$ collisions at $\sqrt{s_{N N}}=200 \mathrm{GeV}$, Phys. Rev. C 91, 031901 (2015).

[62] J. R. Batley et al. (NA48/2 Collaboration), Search for the dark photon in $\pi^{0}$ decays, Phys. Lett. B 746, 178 (2015).

[63] R. Essig, P. Schuster, N. Toro, and B. Wojtsekhowski, An electron fixed target experiment to search for a new vector boson $\mathrm{A}^{\prime}$ decaying to $e^{+} e^{-}$, J. High Energy Phys. 02 (2011) 009.

[64] M. Freytsis, G. Ovanesyan, and J. Thaler, Dark force detection in low energy ep collisions, J. High Energy Phys. 01 (2010) 111.

[65] J. Balewski et al., DarkLight: A search for dark forces at the Jefferson Laboratory Free-Electron Laser facility, arXiv:1307.4432.

[66] B. Wojtsekhowski, D. Nikolenko, and I. Rachek, Searching for a new force at VEPP-3, arXiv:1207.5089.

[67] T. Beranek, H. Merkel, and M. Vanderhaeghen, Theoretical framework to analyze searches for hidden light gauge bosons in electron scattering fixed target experiments, Phys. Rev. D 88, 015032 (2013).

[68] M. Raggi and V. Kozhuharov, Proposal to search for a dark photon in positron on target collisions at DAФNE linac, Adv. High Energy Phys. 2014, 959802 (2014).

[69] B. Echenard, R. Essig, and Yi-M. Zhong, Projections for dark photon searches at Mu3e, J. High Energy Phys. 01 (2015) 113.

[70] M. Battaglieri et al., The Heavy Photon Search test detector, Nucl. Instrum. Methods Phys. Res., Sect. A 777, 91 (2015).

[71] S. Alekhin et al., A facility to search for hidden particles at the CERN SPS: The SHiP physics case, Rep. Prog. Phys. 79, 124201 (2016).

[72] P. Ilten, J. Thaler, M. Williams, and W. Xue, Dark photons from charm mesons at LHCb, Phys. Rev. D 92, 115017 (2015).

[73] D. Curtin, R. Essig, S. Gori, and J. Shelton, Illuminating dark photons with high-energy colliders, J. High Energy Phys. 02 (2015) 157.

[74] M. He, X.-G. He, and C.-K. Huang, Dark photon search at a circular $e^{+} e^{-}$collider, Int. J. Mod. Phys. A 32, 1750138 (2017).

[75] J. Kozaczuk, Dark photons from nuclear transitions, Phys. Rev. D 97, 015014 (2018).

[76] P. Ilten, Y. Soreq, J. Thaler, M. Williams, and W. Xue, Proposed Inclusive Dark Photon Search at LHCb, Phys. Rev. Lett. 116, 251803 (2016). 
[77] J. Feng, I. Galon, F. Kling, and S. Trojanowski, FASER: Forward search experiment at the LHC, Phys. Rev. D 97, 035001 (2018).

[78] J. Alexander, MMAPS: Missing-mass A-prime search, EPJ Web Conf. 142, 01001 (2017).

[79] A. Ariga et al. (FASER Collaboration), FASER's physics reach for long-lived particles, Phys. Rev. D 99, 095011 (2019).

[80] B. Döbrich (NA62 Collaboration), Dark Sectors at fixed targets: The example of NA62, Frascati Phys. Ser. 66, 312 (2018).

[81] P. Agrawal et al., Feebly-Interacting Particles:FIPs 2020 Workshop Report, arXiv:2102.12143.

[82] D. Tucker-Smith and N. Weiner, Inelastic dark matter, Phys. Rev. D 64, 043502 (2001).

[83] J. Bramante, P. J. Fox, G. D. Kribs, and A. Martin, Inelastic frontier: Discovering dark matter at high recoil energy, Phys. Rev. D 94, 115026 (2016).

[84] G. F. Giudice, D. Kim, J.-C. Park, and S. Shin, Inelastic boosted dark matter at direct detection experiments, Phys. Lett. B 780, 543 (2018).

[85] A. Berlin and F. Kling, Inelastic dark matter at the LHC Lifetime Frontier: ATLAS, CMS, LHCb, CODEX-b, FASER, and MATHUSLA, Phys. Rev. D 99, 015021 (2019).

[86] G. Mohlabeng, Revisiting the dark photon explanation of the muon g-2 anomaly, Phys. Rev. D 99, 115001 (2019).

[87] J. Eby, P. J. Fox, R. Harnik, and G. D. Kribs, Luminous signals of inelastic dark matter in large detectors, J. High Energy Phys. 09 (2019) 115.

[88] E. Izaguirre, G. Krnjaic, P. Schuster, and N. Toro, Analyzing the Discovery Potential for Light Dark Matter, Phys. Rev. Lett. 115, 251301 (2015).

[89] J. Alexander et al., Dark sectors 2016 workshop: Community report, arXiv:1608.08632.

[90] T. Moroi, H. Murayama, and M. Yamaguchi, Cosmological constraints on the light stable gravitino, Phys. Lett. B 303, 289 (1993).

[91] T. Asaka, K. Ishiwata, and T. Moroi, Right-handed sneutrino as cold dark matter, Phys. Rev. D 73, 051301 (2006).

[92] M. Shaposhnikov and I. Tkachev, The nuMSM, inflation, and dark matter, Phys. Lett. B 639, 414 (2006).

[93] A. Kusenko, Sterile Neutrinos, Dark Matter, and the Pulsar Velocities in Models with a Higgs Singlet, Phys. Rev. Lett. 97, 241301 (2006).

[94] L. J. Hall, K. Jedamzik, J. March-Russell, and S. M. West, Freeze-in production of FIMP dark matter, J. High Energy Phys. 03 (2010) 080.

[95] D. E. Kaplan, M. A. Luty, and K. M. Zurek, Asymmetric dark matter, Phys. Rev. D 79, 115016 (2009).

[96] G. Krnjaic, Probing light thermal dark-matter with a Higgs portal mediator, Phys. Rev. D 94, 073009 (2016).

[97] M. J. Dolan, F. Kahlhoefer, C. McCabe, and K. SchmidtHoberg, A taste of dark matter: Flavour constraints on pseudoscalar mediators, J. High Energy Phys. 03 (2015) 171; Erratum, J. High Energy Phys. 07 (2015) 103.

[98] Y. Hochberg, E. Kuflik, T. Volansky, and Jay G. Wacker, Mechanism for Thermal Relic Dark Matter of Strongly Interacting Massive Particles, Phys. Rev. Lett. 113, 171301 (2014).

[99] Y. Hochberg, E. Kuflik, and H. Murayama, SIMP Spectroscopy, J. High Energy Phys. 05 (2016) 090.
[100] E. Kuflik, M. Perelstein, N. R.-L. Lorier, and Y.-D. Tsai, Elastically Decoupling Dark Matter, Phys. Rev. Lett. 116, 221302 (2016).

[101] E. Kuflik, M. Perelstein, N. R.-L. Lorier, and Y.-D. Tsai, Phenomenology of ELDER dark matter, J. High Energy Phys. 08 (2017) 078.

[102] Y.-D. Tsai, Robert McGehee, and Hitoshi Murayama, Resonant self-interacting dark matter from dark QCD, arXiv:2008.08608.

[103] P. J. Fitzpatrick, H. Liu, T. R. Slatyer, and Y.-D. Tsai, New pathways to the relic abundance of vector-portal dark matter, arXiv:2011.01240.

[104] M. Pospelov, Secluded U(1) below the weak scale, Phys. Rev. D 80, 095002 (2009).

[105] M. Liu, Direct Search for Dark Photon and Dark Higgs at SeaQuest/E1067, https://indico.fnal.gov/event/13702/ session/9/material/slides/0? contribId=128.

[106] See Supplemental Material at http://link.aps.org/ supplemental/10.1103/PhysRevLett.126.181801 for described the decay and scattering detectors at the high energy intensity frontier, the details of the experiments considered in this paper (including CHARM, NuCal, NA62, SeaQuest/DarkQuest, and LongQuest), the details of the analysis, and new constraints one can derive if we consider choices of parameters that are different from that of the main text.

[107] R. D. Ball et al., Parton distributions with LHC data, Nucl. Phys. B867, 244 (2013).

[108] P. Fayet, Light spin $1 / 2$ or spin 0 dark matter particles, Phys. Rev. D 70, 023514 (2004).

[109] M. Tanabashi et al. (Particle Data Group Collaboration), Review of particle physics, Phys. Rev. D 98, 030001 (2018).

[110] M. Davier, A. Hoecker, B. Malaescu, and Z. Zhang, Reevaluation of the hadronic contributions to the muon g-2 and to alpha(MZ), Eur. Phys. J. C 71, 1515 (2011); Erratum, Eur. Phys. J. C 72, 1874 (2012).

[111] M. Davier, A. Hoecker, B. Malaescu, and Z. Zhang, Reevaluation of the hadronic vacuum polarisation contributions to the Standard Model predictions of the muon $g-2$ and $\alpha\left(m_{Z}^{2}\right)$ using newest hadronic cross-section data, Eur. Phys. J. C 77, 827 (2017).

[112] M. Davier, A. Hoecker, B. Malaescu, and Z. Zhang, A new evaluation of the hadronic vacuum polarisation contributions to the muon anomalous magnetic moment and to $\boldsymbol{\alpha}\left(\mathbf{m}_{\mathbf{Z}}^{\mathbf{2}}\right)$, Eur. Phys. J. C 80, 241 (2020).

[113] T. Blum, P. A. Boyle, V. Gülpers, T. Izubuchi, L. Jin, C. Jung, A. Jüttner, C. Lehner, A. Portelli, and J. T. Tsang (RBC, UKQCD Collaborations), Calculation of the Hadronic Vacuum Polarization Contribution to the Muon Anomalous Magnetic Moment, Phys. Rev. Lett. 121, 022003 (2018).

[114] T. Izubuchi, Y. Kuramashi, C. Lehner, and E. Shintani (PACS Collaboration), Finite-volume correction on the hadronic vacuum polarization contribution to the muon $\mathrm{g}-2$ in lattice QCD, Phys. Rev. D 98, 054505 (2018).

[115] A. Keshavarzi, D. Nomura, and T. Teubner, Muon $g-2$ and $\alpha\left(M_{Z}^{2}\right)$ : A new data-based analysis, Phys. Rev. D 97 , 114025 (2018). 
[116] J. Grange et al. (Muon g-2 Collaboration), Muon (g-2) technical design report, arXiv:1501.06858.

[117] M. Abe et al., A new approach for measuring the muon anomalous magnetic moment and electric dipole moment, Prog. Theor. Exp. Phys. 2019, 053C02 (2019).

[118] D. Banerjee et al. (NA64 Collaboration), Search for Invisible Decays of sub-GeV Dark Photons in MissingEnergy Events at the CERN SPS, Phys. Rev. Lett. 118, 011802 (2017).

[119] J. P. Lees et al. (BABAR Collaboration), Search for Invisible Decays of a Dark Photon Produced in $e^{+} e^{-}$Collisions at BABAR, Phys. Rev. Lett. 119, 131804 (2017).

[120] D. Banerjee et al., Dark matter search in missing energy events with NA64, Phys. Rev. Lett. 123, 121801 (2019).

[121] B. Döbrich, J. Jaeckel, and T. Spadaro, Light in the beam dump. Axion-Like Particle production from decay photons in proton beam-dumps, J. High Energy Phys. 05 (2019) 213.

[122] A. Berlin, N. Blinov, G. Krnjaic, P. Schuster, and N. Toro, Dark matter, millicharges, axion and scalar particles, gauge bosons, and other new physics with LDMX, Phys. Rev. D 99, 075001 (2019).

[123] T. Åkesson et al. (LDMX Collaboration), Light dark matter experiment (LDMX), arXiv:1808.05219.

[124] J. R. Jordan, Y. Kahn, G. Krnjaic, M. Moschella, and J. Spitz, Signatures of pseudo-dirac dark matter at highintensity neutrino experiments, Phys. Rev. D 98, 075020 (2018).

[125] J. P. Chou, D. Curtin, and H. J. Lubatti, New detectors to explore the lifetime frontier, Phys. Lett. B 767, 29 (2017).

[126] V. V. Gligorov, S. Knapen, M. Papucci, and D. J. Robinson, Searching for long-lived particles: A compact detector for exotics at LHCb, Phys. Rev. D 97, 015023 (2018).

[127] A. Pierce, B. Shakya, Y. Tsai, and Y. Zhao, Searching for confining hidden valleys at LHCb, ATLAS, and CMS, Phys. Rev. D 97, 095033 (2018).

[128] W. Altmannshofer et al. (Belle-II Collaboration), The Belle II physics book, Prog. Theor. Exp. Phys. 2019, 123C01 (2019).

[129] E. Izaguirre, G. Krnjaic, and B. Shuve, Discovering inelastic thermal-relic dark matter at colliders, Phys. Rev. D 93, 063523 (2016).

[130] J. Liu, Z. Liu, and L.-T. Wang, Enhancing Long-Lived Particles Searches at the LHC with Precision Timing Information, Phys. Rev. Lett. 122, 131801 (2019).

[131] A. Faessler, M. I. Krivoruchenko, and B. V. Martemyanov, Once more on electromagnetic form factors of nucleons in extended vector meson dominance model, Phys. Rev. C 82, 038201 (2010).

[132] L. B. Auerbach et al. (LSND Collaboration), Measurement of electron-neutrino-electron elastic scattering, Phys. Rev. D 63, 112001 (2001).

[133] P. Chung et al. (E895 Collaboration), Near Threshold Production of the Multistrange $\Xi^{-}$Hyperon, Phys. Rev. Lett. 91, 202301 (2003).

[134] B. Aubert et al. (BABAR Collaboration), Search for invisible decays of a light scalar in radiative transitions $v_{3 S} \rightarrow \gamma \mathrm{A} 0$, in Proceedings, 34th International Conference on High Energy Physics (ICHEP 2008): Philadelphia, Pennsylvania, July 30-August 5, 2008(2008) [arXiv:0808.0017].
[135] A. Hook, E. Izaguirre, and J. G. Wacker, Model independent bounds on kinetic mixing, Adv. High Energy Phys. 2011, 859762 (2011).

[136] B. Batell, R. Essig, and Z. Surujon, Strong Constraints on Sub-GeV Dark Sectors from SLAC Beam Dump E137, Phys. Rev. Lett. 113, 171802 (2014).

[137] A. A. Aguilar-Arevalo et al. (MiniBooNE Collaboration), Dark Matter Search in a Proton Beam Dump with MiniBooNE, Phys. Rev. Lett. 118, 221803 (2017).

[138] Céline Boehm, M. J. Dolan, and C. McCabe, A lower bound on the mass of cold thermal dark matter from planck, J. Cosmol. Astropart. Phys. 08 (2013) 041.

[139] D. Green et al., Messengers from the early universe: Cosmic neutrinos and other light relics, Bull. Am. Astron. Soc. 51, 159 (2019).

[140] L. Aliaga et al. (MINERvA Collaboration), Design, calibration, and performance of the MINERvA detector, Nucl. Instrum. Methods Phys. Res., Sect. A 743, 130 (2014).

[141] P. A. N. Machado, O. Palamara, and D. W. Schmitz, The short-baseline neutrino program at Fermilab, Annu. Rev. Nucl. Part. Sci. 69, 363 (2019).

[142] R. Acciarri et al. (MicroBooNE Collaboration), Design and construction of the MicroBooNE detector, J. Instrum. 12, P02017 (2017).

[143] B. Abi et al. (DUNE Collaboration), The DUNE far detector interim design report volume 1: Physics, technology and strategies, arXiv:1807.10334.

[144] F. Bergsma et al. (CHARM Collaboration), A search for decays of heavy neutrinos, Phys. Lett. 128B, 361 (1983).

[145] P. deNiverville, H.-S. Lee, and M.-S. Seo, Implications of the dark axion portal for the muon g-2, B-factories, fixed target neutrino experiments and beam dumps, Phys. Rev. D 98, 115011 (2018).

[146] J. Dorenbosch et al. (CHARM Collaboration), Calibration of the charm fine grained calorimeter, Nucl. Instrum. Methods Phys. Res., Sect. A 253, 203 (1987).

[147] E. Cortina Gil et al. (NA62 Collaboration), The Beam and detector of the NA62 experiment at CERN, J. Instrum. 12, P05025 (2017).

[148] C.-W. Chiang and P.-Y. Tseng, Probing a dark photon using rare leptonic kaon and pion decays, Phys. Lett. B 767, 289 (2017).

[149] E. Cortina Gil et al. (NA62 Collaboration), Search for production of an invisible dark photon in $\pi^{0}$ decays, J. High Energy Phys. 05 (2019) 182.

[150] V. Shiltsev, Fermilab proton accelerator complex status and improvement plans, Mod. Phys. Lett. A 32, 1730012 (2017).

[151] T. Pierog, Iu. Karpenko, J. M. Katzy, E. Yatsenko, and K. Werner, EPOS LHC: Test of collective hadronization with data measured at the CERN Large Hadron Collider, Phys. Rev. C 92, 034906 (2015).

[152] E. Amaldi et al., Inclusive eta production in P P Collision at isr energies, Nucl. Phys. B158, 1 (1979).

[153] K. Jaeger, J. Campbell, G. Charlton, D. Swanson, C. Fu, H. A. Rubin, R. G. Glasser, D. Koetke, and J. Whitmore, Inclusive $\Gamma, \pi^{0}, K^{0}$ and $\Lambda$ production in $12.4-\mathrm{GeV} / c \mathrm{p} \mathrm{p}$ interactions, Phys. Rev. D 11, 1756 (1975). 
[154] M. Bonesini, A. Marchionni, F. Pietropaolo, and T. Tabarelli de Fatis, On Particle production for high-energy neutrino beams, Eur. Phys. J. C 20, 13 (2001).

[155] D. Gorbunov, A. Makarov, and I. Timiryasov, Decaying light particles in the SHiP experiment: Signal rate estimates for hidden photons, Phys. Rev. D 91, 035027 (2015).

[156] Y. Akiba et al., The PHENIX ring imaging Cherenkov detector, Nucl. Instrum. Methods Phys. Res., Sect. A 453, 279 (2000).

[157] Y.-D. Tsai et al., FerMINI: Fermilab search for minicharged particles, Letter of intent in response to DOE RFP "Dark Matter New Initiatives DE-FOA-0002112" (2019).
[158] S. Lim, Highlight on trigger upgrade for dark photon search at SeaQuest (Fermilab), https://www.phenix.bnl .gov/WWW/publish/shlim/E906_DP/dp_trigger_status_ 20170411.pdf.

[159] N. Tran et al., Search for dark sectors with the DarkQuest experiment at Fermilab, DOE Proposal, Report No. LAB 19-2112.

[160] C Baus, T Pierog, and R Ulrich, CRMC, https://web.ikp.kit .edu/rulrich/crmc.html, Accessed: 2019-03-12.

[161] B. Azmoun et al., Conceptual Design Report on a HBD Upgrade for the PHENIX Detector, https://www.phenix .bnl.gov/WWW/TPCHBD/HBD_CDR.pdf. 